\title{
284 DEVELOPMENTAL CHANGES IN EXPRESSION OF PLURIPOTENT GENES IN EARLY EQUINE EMBRYOS
}

\author{
D. B. B. P. Paris ${ }^{\text {A }, ~ E . ~ W . ~ K u i j k ~}{ }^{\text {B }}$, B. A. J. Roelen ${ }^{\text {B }}$ and T. A. E. Stout ${ }^{A}$ \\ A Department of Equine Sciences, Utrecht University, Utrecht, the Netherlands; \\ B Department of Farm Animal Health, Utrecht University, Utrecht, the Netherlands
}

\begin{abstract}
Genes involved in maintaining pluripotency have potential use in establishing cell lines for regenerative medicine. However, the genes differ subtly between species, and are poorly described in the horse. In this study we examined changes in expression of pluripotency-associated genes in horse embryos during blastocyst formation. Twenty-one grade 1-2 embryos where recovered from mares by uterine lavage on Day 6-7 after ovulation. Embryos were classified by developmental stage (morula, early or expanded blastocyst: $n$ $=5,7$ and 9, respectively) and their diameter measured by micrometer, before being snap frozen. Subsequently, mRNA from individual embryos was extracted, DNAse-treated and synthesized into cDNA using an AllPrep Mini Kit and Superscript III Reverse Transcriptase (Qiagen, Venlo, and Invitrogen, Breda respectively, the Netherlands). Equine-specific intron-spanning/overlapping primers were designed using PerlPrimer v1.1.14 by BLAST searching the NCBI horse genome for 5 genes associated with pluripotency in other species (octamer binding protein OCT4, transcription factor NANOG, developmental pluripotencyassociated DPPA4, growth and differentiation factor GDF3 and telomerase reverse transcriptase TERT) and 2 reference genes (signal recognition particle SRP14 and phosphoglycerate kinase PGK1). Relative gene expression was then examined by quantitative PCR using an iQ5 RT PCR Detection System (BioRad, Veenendaal, the Netherlands). Relationships were tested by Pearson correlations and differences between developmental stages were tested by ANCOVA. Embryos ranged in diameter from 126 to $680 \mu \mathrm{m}$. As expected, absolute expression of all pluripotency markers increased with increasing embryo diameter $(P=$ $0.000 ; R=0.93,0.92,0.88,0.86$ and 0.76 for NANOG, DPPA4, GDF3, OCT4 and TERT, respectively). After normalization with SRP14 and PGK1, significant negative correlations with embryo diameter were apparent for OCT4, NANOG and DPPA4 $(P<0.001 ; R=-0.73,-0.69$ and -0.53 , respectively). Moreover, all 5 pluripotency genes were down-regulated as embryonic development progressed $(P<0.05)$, although the time-course differed between genes. The DPPA4 and OCT4 expression decreased significantly at both the morula-early blastocyst and early-expanded blastocyst transitions, whereas NANOG expression only decreased significantly between the early-expanded blastocyst stages and GDF3 and TERT expression only between the morula-early blastocyst stages. Down-regulation of pluripotent gene expression during early development is consistent with increased cohorts of cells differentiating into trophectoderm and primitive endoderm, leaving an ever decreasing proportion of pluripotent cells in the inner cell mass. Furthermore, the different time courses of down-regulation may reflect different roles of the examined genes in developmental processes. For example, early and continued down-regulation of OCT4 is consistent with a role in differentiation between ICM and trophectoderm, whereas the slightly later onset of NANOG down-regulation better fits a role in determining between pluripotent epiblast and primitive endoderm, as described in other species.
\end{abstract}

Reproduction, Fertility and Development 21(1) 239-239

Published: 09 December 2008

doi: 10.1071/RDv21n1Ab284

(c) IETS 20092009

Printed from CSI RO PUBLI SHI NG - Reproduction, Fertility and Development

http://www.publish.csiro. au/view/journals/dsp_journal_fulltext.cfm?nid=44\&f=RDv21n1Ab284 